Canad. Math. Bull. Vol. 21 (1), 1979

\title{
TOURNAMENTS WHOSE SUBTOURNAMENTS ARE IRREDUCIBLE OR TRANSITIVE
}

\author{
BY \\ J. W. MOON
}

\begin{abstract}
Beineke and Harary gave an example of a family of tournaments $T_{n}$ such that every subtournament of $T_{n}$ is irreducible or transitive. We characterize all tournaments with this property.
\end{abstract}

1. Introduction. A tournament $T_{n}$ consists of a finite set of nodes $1,2, \ldots, n$ such that each pair of distinct nodes $i$ and $j$ is joined by exactly one of the arcs $\overrightarrow{i j}$ or $\overrightarrow{j i}$. If the arc $\overrightarrow{i j}$ is in $T_{n}$ we say that $i$ beats $j$ or $j$ loses to $i$ and write $i \rightarrow j$. If each node of a subtournament $A$ beats each node of a subtournament $B$, we write $A \rightarrow B$ and let $A+B$ denote the subtournament determined by the nodes of $A$ and $B$.

A tournament $T_{n}$ is reducible if it can be expressed as $T_{n}=A+B$ for some non-empty tournaments $A$ and $B$; otherwise it is irreducible. A tournament is transitive if there exists a linear ordering of its nodes such that $i \rightarrow j$ if and only if $i$ precedes $j$ in the ordering. (Notice that the trivial tournament $T_{1}$ is the only tournament that is both transitive and irreducible.) A tournament $T_{n}$ is highly-regular if $n$ is odd and there exists a cyclic ordering of the nodes such that $i \rightarrow j$ if and only if $j$ is one of the first $\frac{1}{2}(n-1)$ successors of $i$ in the ordering; we remark that the ordering with this property is unique. (These tournaments were introduced by Kendall and Babington Smith [3]. For additional material on tournaments in general, see [2] and [4].)

We say a tournament $T_{n}$ has property $\mathscr{L}$ if every subtournament of $T_{n}$ is irreducible or transitive. Beineke and Harary [1] showed that highly-regular tournaments have property $\mathscr{L}$. Our main object here is to establish a structural characterization of all tournaments with property $\mathscr{L}$. Before stating this characterization we need to introduce some additional terminology.

2. Statement of characterization. We say the nodes in a subtournament $A$ of $T_{n}$ are equivalent if for any node $q$ not in $A$ either $q \rightarrow A$ or $A \rightarrow q$. (Equivalent nodes are sometimes said to form a convex subset; see, e.g., [6]). Suppose the nodes of $T_{n}$ are partitioned into disjoint subtournaments $E_{1}, \ldots, E_{m}$ of equivalent nodes, where the subscripts $1, \ldots, m$ serve merely to distinguish between different subtournaments. Then $E_{i} \rightarrow E_{j}$ or $E_{j} \rightarrow E_{i}$ for

Received by the editors January 4, 1978. 
$1 \leq i, j \leq m$. If $R_{m}$ denotes the tournament on $m$ nodes in which $i \rightarrow j$ if and only if $E_{i} \rightarrow E_{j}$, then we write $T_{n}=R_{m}\left(E_{1}, \ldots, E_{m}\right)$. (If the subtournaments $E_{1}, \ldots, E_{m}$ are isomorphic, then $T_{n}$ is the composition of $R_{m}$ with $E_{1}$; see [4; p.78].) A tournament $T_{n}$ is simple (see, e.g., [2] and [6]) if it has no non-trivial proper subtournaments of equivalent nodes, that is, if the equation $T_{n}=$ $R_{m}\left(E_{1}, \ldots, E_{m}\right)$ implies that $m=1$ and $E_{1}=T_{n}$ or that $m=n, T_{n}=R_{m}$, and $E_{i}=T_{1}$ for each $i$. We can now state our main result.

THEOREM 1. A tournament $T_{n}$ has property $\mathscr{L}$ if and only if

$$
T_{n}=R_{m}\left(E_{1}, \ldots, E_{m}\right)
$$

where $R_{m}$ is a highly-regular tournament and the subtournaments $E_{1}, \ldots, E_{m}$ all are transitive.

3. Two preliminary results. For any node $i$ in a tournament $T_{n}$, let $\Gamma(i)$ and $\Gamma^{-1}(i)$ denote the subtournaments determined by the nodes of $T_{n}$ that lose to $i$ and the nodes that beat $i$, respectively. It may be that $\Gamma(i)$ or $\Gamma^{-1}(i)$ is the empty tournament. We shall use the following lemmas in the proof of Theorem 1.

LeMMA 1. A tournament $T_{n}$ has property $\mathscr{L}$ if and only if $\Gamma(i)$ and $\Gamma^{-1}(i)$ are empty or transitive for all nodes $i$ of $T_{n}$.

Proof. If $\Gamma(i)$ or $\Gamma^{-1}(i)$ is non-empty and non-transitive for some node $i$ of $T_{n}$, then the corresponding subtournament $i+\Gamma(i)$ or $\Gamma^{-1}(i)+i$ is neither irreducible nor transitive and, consequently, $T_{n}$ does not have property $\mathscr{L}$. Conversely, if $T_{n}$ does not have property $\mathscr{L}$, then it contains a reducible non-transitive subtournament $S=A+B$ where $A$ and $B$ are both non-empty and at least one of them is non-transitive. Let $i$ and $j$ denote any nodes in $A$ and $B$, respectively. If $B$ is non-transitive then $\Gamma(i)$ is neither empty nor transitive, and if $A$ is non-transitive then $\Gamma^{-1}(j)$ is neither empty nor transitive. This completes the proof of Lemma 1.

LeMma 2. A non-simple tournament $T_{n}=R_{m}\left(E_{1}, \ldots, E_{m}\right)$, where $1<m<n$, has property $\mathscr{L}$ if and only if the tournament $R_{m}$ has property $\mathscr{L}$ and the subtournaments $E_{1}, \ldots, E_{m}$ all are transitive.

Proof. The sufficiency of the conditions follows readily from Lemma 1 and the necessity of the condition that $R_{m}$ must have property $\mathscr{L}$ is obvious. Suppose some subtournament $E_{i}$ is not transitive. If $j$ is any node of $T_{n}$ not in $E_{i}$, and such a node exists since $m>1$, then either $j \rightarrow E_{i}$ or $E_{i} \rightarrow j$. If $j \rightarrow E_{i}$ then $\Gamma(j)$ is non-empty and non-transitive. Thus the conditions are also necessary, in view of Lemma 1. This completes the proof of Lemma 2.

4. Proof of theorem 1. All highly-regular tournaments $T_{n}$ have property $\mathscr{L}$, as was shown in [1], since they clearly satisfy the condition of Lemma 1 . Thus 
the tournaments described in the statement of Theorem 1 certainly have property $\mathscr{L}$, by Lemma 2 .

Let $T_{n}$ denote any tournament with property $\mathscr{L}$. We may assume that $T_{n}$ is non-transitive and simple, in view of Lemma 2, and we may also suppose that $n \geq 3$. To complete the proof of Theorem 1 we must show that $T_{n}$ is highlyregular.

The score of any node $i$ is the number of nodes beaten by $i$. If $s$ denotes the maximum of the scores of nodes of $T_{n}$, then $1 \leq \frac{1}{2}(n-1) \leq s$; furthermore, $s \leq n-2$ since if $s=n-1$ then $T_{n}$ would be reducible and not simple. Let $x$ denote any node with score $s$. We may suppose, for convenience, that $x$ has label $n$; that the nodes of $\Gamma(n)$ are labelled $1,2, \ldots, s$; and that the nodes of $\Gamma^{-1}(n)$ are labelled $s+1, \ldots, n-1$. Since $T_{n}$ has property $\mathscr{L}$ it follows that $\Gamma(n)$ and $\Gamma^{-1}(n)$ are both transitive, by Lemma 1 . So we may further assume that if $1 \leq i<j \leq s$, then $i \rightarrow j$ in $\Gamma(n)$; and that if $s+1 \leq u<v \leq n-1$, then $u \rightarrow v$ in $\Gamma^{-1}(n)$. This labelling defines a natural circular ordering of the nodes of $T_{n}$ and, in what follows, when we refer to the successors or predecessors of a node, we mean the successors or predecessors with respect to this ordering. At this stage we may say that node $n$ beats its first $s$ successors (and loses to its $n-1-s$ predecessors) and that each node in $\Gamma(n)$ and $\Gamma^{-1}(n)$ beats its immediate successors in these subtournaments (and loses to its immediate predecessors in these subtournaments). We want to show that the same is true for each node $i$.

Suppose for some node $x$ in $\Gamma(n)$ there exist two nodes $u$ and $v$ in $\Gamma^{-1}(n)$, where $u<v$, such that $u \rightarrow x$ and $x \rightarrow v$. Then $v \rightarrow n, n \rightarrow x$, and $x \rightarrow v$; that is, the nodes $v, n$, and $x$ form a 3-cycle, and all three of these nodes lose to $u$. If this were the case, then $\Gamma(u)$ would be neither empty nor transitive and $T_{n}$ would not have property $\mathscr{L}$. It follows, therefore, that if a node $x$ in $\Gamma(n)$ beats any nodes of $\Gamma^{-1}(n)$, then those nodes form a subset of consecutive nodes of $\Gamma^{-1}(n)$ starting with node $s+1$.

Node 1 beats the $s-1$ nodes $2, \ldots, s$ and, in addition to these nodes, it beats just one node of $\Gamma^{-1}(n)$. For, if node 1 beats more than one node of $\Gamma^{-1}(n)$, its score would exceed the maximum score $s$; and, if node 1 lost to all the nodes of $\Gamma^{-1}(n)$, nodes 1 and $n$ would be equivalent, contradicting the assumption that $T_{n}$ is simple. Therefore, node 1 beats one node of $\Gamma^{-1}(n)$, namely, node $s+1$, and loses to the remaining nodes of $\Gamma^{-1}(n)$.

We next observe that node $s$ must beat node $s+1$, for otherwise it would have score zero and $T_{n}$ would be reducible and not simple. Finally, we assert that all remaining nodes of $\Gamma(n)$ must also beat node $s+1$. For, if there were some node $x$, where $1<x<s$, such that $s+1 \rightarrow x$, the nodes $x$, $s$, and $s+1$ would form a 3 -cycle in $\Gamma(1)$; then $\Gamma(1)$ would be neither empty nor transitive and $T_{n}$ would not have property $\mathscr{L}$.

It follows form the preceding observations that node 1 beats its first $s$ 
successors (and loses to its $n-1-s$ predecessors) and that each node of $\Gamma$ (1) and $\Gamma^{-1}(1)$ beats its immediate successors in these subtournaments (and loses to its immediate predecessors in these subtournaments). By repeating this argument we find that the same is true for every node $i$ of $T_{n}$. In particular, each node of $T_{n}$ beats its $s$ immediate successors. This implies that $s=\frac{1}{2}(n-1)$ and that $n$ is odd. Hence, $T_{n}$ is highly-regular by definition. This completes the proof of Theorem 1 .

5. Enumerating tournaments with property $\mathscr{L}$. Let $f(n)$ denote the number of tournaments $T_{n}$ with $n$ labelled nodes that have property $\mathscr{L}$, and let $g(n)$ denote the corresponding number when the labels of the nodes are not taken into account.

THEOREM 2. If $n=1,2, \ldots$, then

$$
f(n)=(n-1) ! 2^{n-1}
$$

and

$$
g(n)=\frac{1}{2 n} \sum_{k} \phi(k) 2^{n / k}
$$

where $\phi(k)$ denotes the Euler $\phi$-function and the sum is over all odd divisors $k$ of $n$.

Proof. Let $T_{n}=R_{m}\left(E_{1}, \ldots, E_{m}\right)$ denote a tournament with property $\mathscr{L}$, where $R_{m}$ is highly-regular. It follows from the definition of $R_{m}$ that there exists a circular ordering of the subtournaments $E_{1}, \ldots, E_{m}$ such that $E_{i} \rightarrow E_{i}$ if and only if $E_{j}$ is one of the first $\frac{1}{2}(m-1)$ successors of $E_{i}$ with respect to this ordering (and the ordering with this property is unique). Furthermore, there is a unique linear ordering of the nodes in each transitive subtournament $E_{i}$ such that each node $u$ in $E_{i}$ beats its successors with respect to the ordering in $E_{i}$. These orderings, that is, the circular ordering of the subtournaments $E_{i}$ and the linear orderings of the nodes in the individual subtournaments $E_{i}$, induce a circular ordering of the $n$ nodes of $T_{n}$ such that each node $u$ beats its immediate successors that belong to the same or one of the next $\frac{1}{2}(m-1)$ subtournaments $E_{i}$.

It follows, therefore, that tirere is a one-to-one correspondence between the labelled tournaments $T_{n}$ with property $\mathscr{L}$ and the circular arrangements of $m$ 0 's and the $n$ numbers $1,2, \ldots, n$ such that no two 0 's are next to each other. The numbers $1,2, \ldots, n$ correspond to the nodes of $T_{n}$ and $i \rightarrow j$ in $T_{n}$ if and only if there are at most $\frac{1}{2}(m-1) 0$ 's between $i$ and $j$ in the circular arrangement. There are $(n-1)$ ! circular arrangements of the numbers $1,2, \ldots, n$ and for each such arrangement there are $\left(\begin{array}{c}n \\ m\end{array}\right)$ ways to insert $m 0$ 's. Since $m$ can be 
any odd number not exceeding $n$, it follows that

$$
f(n)=(n-1) !\left\{\left(\begin{array}{l}
n \\
1
\end{array}\right)+\left(\begin{array}{l}
n \\
3
\end{array}\right)+\cdots\right\}=(n-1) ! 2^{n-1},
$$

as required.

Now suppose the labels of the nodes are not taken into account. It is not difficult to see that there is a one-to-one correspondence between the unlabelled tournaments $T_{n}=R_{m}\left(E_{1}, \ldots, E_{m}\right)$ with property $\mathscr{L}$ and the circular arrangements of $n$ 1's and $m$ 0's such that no two 0's are next to each other. (Two such arrangements are considered the same if the differ only by a rotation.) If no two 0 's are next to each other, then each 0 is followed by a 1 ; thus, the circular arrangements of $n 1$ 's and $m$ 0's with no two 0 's next to each other are equinumerous with the circular arrangements of $(n-m) 1$ 's and $m$ 0 's. It is well-known (see, e.g., [5; p.162]) that the number of such arrangements is

$$
\frac{1}{n} \sum_{k} \phi(k)\left(\begin{array}{c}
n / k \\
m / k
\end{array}\right)
$$

where the sum is over all divisors $k$ of $m$ and $n$. When we sum this expression over all odd numbers $m$ not exceeding $n$ we obtain the required formula for $g(n)$. (Since $m$ is odd it follows that $k$ is odd.)

Notice that when $n$ is a power of 2 , then

$$
g(n)=\frac{1}{n} 2^{n-1}
$$

and when $n$ is an odd prime $p$, then

$$
g(p)=\frac{1}{p}\left(2^{p-1}+p-1\right)
$$

The preparation of this paper was assisted by a grant from the National Research Council of Canada.

\section{REFERENCES}

1. L. W. Beineke and F. Harary, The maximum number of strongly connected subtournaments, Canad. Math. Bull. 8 (1965), 491-498.

2. L. W. Beineke and R. J. Wilson, A survey of recent results on tournaments, Recent Advances in Graph Theory, Academia Praha, 1975, 31-48.

3. M. G. Kendall and B. Babington Smith, On the method of paired comparisons, Biometrika 31 (1940), 324-345.

4. J. W. Moon, Topics on Tournaments, Holt, Rinehart, and Winston, New York, 1968.

5. J. Riordan, An Introduction to Combinatorial Analysis, Wiley, New York, 1958.

6. J. C. Varlet, Convexity in tournaments, Bull. Soc. Roy. Sci. Liège, 45 (1976), 570-586.

DepartMent OF MATHEMATICS

UNIVERSITY OF THE WITWATERSRAND

JohanNesburg 2001 SOUTH AFricA 